\title{
IL-7 fine-tunes T-cell homeostasis
}

The survival of naive $\mathrm{T}$ cells depends on both T-cell receptor (TCR) and cytokine signals. In particular, interleukin-7 (IL-7) has been shown to be an essential survival factor in combination with TCR signalling. It was assumed that the homeostatic proliferation of naive $\mathrm{T}$ cells in lymphopaenic hosts follows the same rules, but Seddon and Zamoyska have shown that IL-7 can induce proliferation independently and that the relative contribution of IL-7 signalling to TCR-induced proliferation fine-tunes the response to different conditions.

The authors used $\mathrm{T}$ cells from $\mathrm{Lck} 1^{\text {ind }}$ mice, which express a tetracycline-inducible $L c k$ transgene targeted to T cells on an Lck-knockout background. Previous studies have shown that Lck is essential to transduce T-cell receptor (TCR)-mediated homeostatic proliferation. The expression of Lck by these $\mathrm{T}$ cells can be induced by administration of the tetracycline derivative dox.

CFSE-labelled $\mathrm{T}$ cells from $\mathrm{Lck} 1^{\text {ind }}$ mice were transferred to recipients that lack expression of both Lck and Fyn ( $L c k^{\text {neg }} F y n^{\text {neg }}$ hosts), which have a complete block in thymic development and, therefore, no peripheral T cells. As expected, wild-type and Lck $1^{\text {ind }} \mathrm{T}$ cells transferred to doxfed hosts had similar patterns of proliferation. But surprisingly, in the absence of Lck expression (dox-free hosts), $\mathrm{T}$ cells from $\mathrm{Lck} 1^{\text {ind }}$ mice still underwent marked proliferation. This indicates that homeostatic proliferation in lymphopaenic hosts can occur in the complete absence of TCR-mediated signals. This was confirmed by the proliferation of Lck-deficient $\mathrm{T}$ cells in hosts lacking MHC class II molecules.

Could this Lck-independent proliferation of T cells be mediated by IL-7? This was tested by treating recipient mice with an antibody that inhibits signalling by the IL-7 receptor (IL-7R). The proliferation of Lck $1^{\text {ind }} \mathrm{T}$ cells in dox-free $L c k^{\text {neg }} F y n^{\text {neg }}$ hosts was blocked completely by this antibody, which indicates that homeostatic proliferation is mediated entirely by signals through the TCR and IL-7R. Furthermore, these signals can operate independently to induce proliferation.

But, how do these two pathways interact to control proliferation under different conditions? The proliferation of wild-type T cells correlates with the extent of T-cell deficiency in the recipient. However, the proliferation of T cells lacking Lck is far more sensitive to the presence of host cells and occurs only in the most lymphopaenic hosts. So, the signalling requirements for proliferation are different from those for survival (see figure). In a full host, both IL-7 and TCR signals are required for naive $\mathrm{T}$-cell survival, whereas in

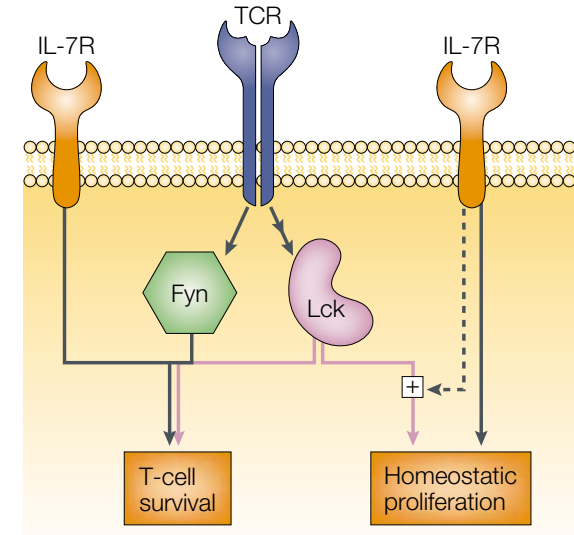

(01992. The American Association of Immunologists, Inc.

extreme lymphopaenia, either IL-7 or TCR signals can induce proliferation directly. And somewhere in the middle (mild lymphopaenia), the two signals synergize to induce an appropriate response.

Kirsty Minton

(2) References and links

ORIGINAL RESEARCH PAPER Seddon, B. \& Zamoyska, R TCR and IL-7 receptor signals can operate independently or synergize to promote lymphopenia-induced expansion of naive T cells. J. Immunol. 169, 3752-3759 (2002)

FURTHer READing Tuma, R. A. \& Pamer, E. G. Homeostasis of naive, effector and memory CD8 T cells. Curr. Opin. Immunol. 14, 348-353 (2002)

\section{NATURAL KILLER CELLS}

\section{On guard}

Like armed guards, natural killer (NK) cells are ready to respond immediately to attack. The constitutive expression of perforin, which facilitates the delivery of apoptosisinducing granzymes to target cells, is essential for this state of readiness. But, little is known about how perforin gene expression is regulated. Now, a study in Immunity shows that the transcription factor MEF, which is encoded by a gene located on human chromosome Xq26, is essential for the expression of perforin by NK cells, and it provides clues to the basis of a human genetic disease.

ETS-family members have been shown previously to regulate the expression of perforin, but the specific proteins involved have not been identified. ETS1 and MEF are possible candidates. In this study, Lacorazza and co-workers generated Mefdeficient mice. Similar to Ets 1deficient mice, $\mathrm{T}$ - and B-cell development were normal, but the number of NK cells was reduced markedly in the Mefdeficient mice. Bonemarrow-transfer experiments showed that this was owing to a developmental defect, rather than to an abnormal microenvironment. So, MEF and ETS1 might work together to promote development of the NK-cell lineage.
In addition, $M e f^{-/-} \mathrm{NK}$ cells could not kill tumour-cell targets and contained little or no perforin, even after activation. However, perforin expression was induced normally in $\mathrm{CD8}^{+} \mathrm{T}$ cells, which indicates that perforin expression is controlled by different mechanisms in NK cells and $\mathrm{CD}^{+} \mathrm{T}$ cells. Experiments with a reporter gene construct confirmed that the perforin promoter is activated strongly by Mef, but not by Ets 1 . As Mef-deficient mice age, they develop perivascular lymphocytic infiltration, which is similar to the symptoms of familial haemophagocytic lymphohistiocytosis (FHL). Patients with FHL have defective NK cells, and some have mutations in the perforin gene. This study indicates that it could be worth looking for $M E F$ mutations in male patients with FHL.

(0) References and links Jennifer Bell ORIGINAL RESEARCH PAPER Lacorazza, H. D. et al. The ETS protein MEF plays a critical role in perforin gene expression and the development of natural killer and NK-T cells. Immunity 17, 437-449 (2002) 